\title{
BANACH SPACES WITH \\ BIHOLOMORPHICALLY EQUIVALENT UNIT \\ BALLS ARE ISOMORPHIC
}

\author{
WILHELM KAUP AND HARALD UPMEIER
}

\begin{abstract}
It is shown that in every complex Banach space $E$ with open unit ball $D \subset E$ there is a closed $C$-linear subspace $V \subset E$ such that $V \cap D$ is the orbit of the origin $0 \in E$ under the group $\operatorname{Aut}(D)$ of all biholomorphic automorphisms of $D$. In particular two complex Banach spaces are isometrically equivalent if and only if their open unit balls are biholomorphically equivalent.
\end{abstract}

Suppose $E, F$ are complex Banach spaces and $U \subset E$ is an open subset. Then a mapping $f: U \rightarrow F$ is called holomorphic if the Fréchet derivative of $f$ at $x$ (denoted by $f^{\prime}(x)$ ) exists for every $x \in U$. (For details compare [6].) In this paper we show that the Banach space $E$ is completely determined by the holomorphic structure of its (open) unit ball; more precisely,

THEOREM. The complex Banach spaces $E$ and $\tilde{E}$ are isometrically isomorphic if and only if there is a biholomorphic mapping from the unit ball of $E$ onto the unit ball of $\tilde{E}$.

This answers a question L. A. Harris raised in [3, p. 15].

For the proof let $E$ be a fixed complex Banach space with unit ball $D \subset E$. Let $\mathfrak{b}$ denote the set of all holomorphic vector fields on $D$ i.e. of all expressions $X=f(z) \partial / \partial z$ where $f: D \rightarrow E$ is a holomorphic mapping and $z$ denotes the coordinate of $D$. Then $\mathfrak{b}$ is a complex Lie algebra in the product

$$
\left[f(z) \frac{\partial}{\partial z}, g(z) \frac{\partial}{\partial z}\right]=\left(g^{\prime}(z) f(z)-f^{\prime}(z) g(z)\right) \frac{\partial}{\partial z} .
$$

For every integer $\nu$ the Lie algebra $\mathfrak{v}$ contains the linear subspace

$$
\mathfrak{P}_{\nu}=\left\{h(z) \frac{\partial}{\partial z} \mid h: E \rightarrow E \text { is a homogeneous polynomial of degree } \nu+1\right\} .
$$

Obviously $\left[\mathfrak{P}_{\nu}, \mathfrak{P}_{\mu}\right] \subset \mathfrak{P}_{\nu+\mu}$ for every $\nu$ and $\mu$.

Since $D$ is a bounded domain in $E$, the set $g$ of all complete holomorphic vector fields on $D$ has the following properties (compare [7]):

(0) Every $h(z) \partial / \partial z \in \mathrm{g}$ is uniquely determined by $h(0)$ and $h^{\prime}(0)$ (Cartan's uniqueness theorem).

Received by the editors October 29, 1975.

AMS (MOS) subject classifications (1970). Primary 32M10, 46B99; Secondary 22E65.

(1) American Mathematical Society 1976 
(1) $\mathfrak{g} \subset \mathfrak{v}$ is a real Lie subalgebra satisfying $\mathfrak{g} \cap i \mathfrak{g}=0$.

(2) For every positive $r<1,\|h(z) \partial / \partial z\|=\sup _{\|z\| \leqslant r}\|h(z)\|$ defines a norm on $g$. Any two of these norms are equivalent and make $g$ a Banach Lie algebra.

\section{Lemma 1. There exists a topological direct sum decomposition}

$$
\mathfrak{g}=\mathfrak{f} \oplus \mathfrak{p},
$$

where $\mathfrak{f}=\mathfrak{g} \cap \mathfrak{P}_{0}, \mathfrak{p}=\mathfrak{g} \cap\left(\mathfrak{P}_{-1} \oplus \mathfrak{P}_{1}\right)$. This decomposition is ad( $\left.\mathfrak{f}\right)$-invariant and, furthermore, $\mathrm{g} \cap \mathfrak{B}_{-1}=\mathrm{g} \cap \mathfrak{P}_{1}=0$.

Proof. The holomorphic vector field $Z:=i \partial / \partial z$ is in $g$ since $D$ is invariant under the corresponding one parameter group $z \mapsto e^{i t} z, t \in \mathbf{R}$. Consider a holomorphic vector field $X \in \mathrm{g}$. Then in a certain neighborhood of the origin, $X$ may be given by a convergent series $X=\sum_{\nu=-1}^{\infty} X_{\nu}$, where $X_{\nu} \in \mathfrak{B}_{\nu}$. Since $\mathrm{g}$ is a real Lie algebra the vector field $Y:=p(\operatorname{ad} Z) X=\sum_{\nu=2}^{\infty} p(i v) X_{\nu}$ is also in $\mathrm{g}$, where the real polynomial $p \in \mathbf{R}[t]$ is given by $p=t\left(t^{2}+1\right)$. Applying Cartan's uniqueness theorem (0), we get $Y=0$, i.e. $X_{\nu}=0$ for $\nu \geqslant 2$, and hence $X=X_{-1}+X_{0}+X_{1}$. But $X_{0}=X+(\operatorname{ad} Z)^{2} X$ is also in $\mathrm{g}$ and the decomposition (3) follows immediately from this. For every $X \in \mathfrak{g} \cap \mathfrak{B}_{1}$ we have $i X=(\operatorname{ad} Z) X \in \mathfrak{g}$ and, hence $\mathfrak{g} \cap \mathfrak{B}_{1} \subset \mathfrak{g} \cap i \mathrm{~g}=0$. In the same way we get $g \cap \mathfrak{P}_{-1}=0$. Q.E.D.

Next consider the R-linear subspace $V=\{f(0) \mid f(z) \partial / \partial z \in \mathfrak{g}\}$ of $E$. Since $\mathfrak{g} \cap \mathfrak{P}_{1}=0$, for every $\alpha \in V$ there is a unique symmetric continuous bilinear mapping $q_{\alpha}: E \times E \rightarrow E$ such that $\left(\alpha-q_{\alpha}(z, z)\right) \partial / \partial z \in \mathrm{g}$.

LEMMA 2. $V \subset E$ is closed.

Proof. Fix a positive $d \leqslant \frac{1}{3}$ and consider holomorphic vector fields $X=h(z) \partial / \partial z \in \mathfrak{p}$ satisfying

$$
\|h(z)\| \leqslant d^{2} / 6 \text { if }\|z\| \leqslant 2 d .
$$

Denote by $L(E)$ the endomorphism algebra of $E$ and endow the Banach space $F=E \times L(E)$ with the max-norm. Consider the one parameter group $g_{t}$ $=\exp (t X)$ associated with $X$ and define $u: \mathbf{R} \rightarrow D \times L(E) \subset F$ by $u(t)$ $=\left(g_{t}(0), g_{t}^{\prime}(0)\right)$. Then there exists a positive $s \leqslant 1$ such that $u(t) \in U$ for all $t$ with $|t| \leqslant s$, where $U \subset F$ is the open ball with radius $d$ and center $u(0)=\left(0, \mathrm{id}_{E}\right)$. Then $u$ satisfies the differential equation

$$
d u / d t=f(u),
$$

where $f: D \times L(E) \rightarrow F$ is defined by $f(x, \lambda)=\left(h(x), h^{\prime}(x) \circ \lambda\right)$. It follows from (4) by a simple computation that $f^{\prime}$ is bounded by 1 on $U \subset D \times L(E)$. Therefore we get

$$
\|u(t)-u(0)\| \leqslant\|f(u(0))\|\left(e^{s}-1\right) \leqslant\|h(0)\| 3 s<s d \text { if }|t| \leqslant s .
$$

(Compare [1, 10.5.1].) In particular we may assume $s=1$. Applying [2, 
Theorem 1] to the function $\delta\left(g_{t}-g_{t}(0)\right)$, where $\delta=\left(1+\left\|g_{t}(0)\right\|\right)^{-1}$, we find a constant $c>0$ such that for $t \in \mathbf{R}$ and $\|z\| \leqslant 2 d$,

$$
\left\|g_{t}(z)-z\right\| \leqslant c\|u(t)-u(0)\|
$$

i.e.

$$
\left\|g_{t}(z)-z\right\| \leqslant 3 c\|h(0)\||t| \quad \text { if }|t| \leqslant 1,\|z\| \leqslant 2 d .
$$

From this we get finally

$$
\|X\|_{i}:=\sup _{\|z\| \leqslant 2 d}\|h(z)\| \leqslant 3 c\|h(0)\|,
$$

where $c$ does not depend on $X$. (Compare [7, Korollar 4.6].) Therefore the continuous, $\mathbf{R}$-linear mapping $\varphi: \mathfrak{p} \rightarrow V, \varphi(h(z) \partial / \partial z)=h(0)$ is bijective and has a continuous inverse, i.e. $V$ is complete. Q.E.D.

Lemma 3. $V$ is a $\mathbf{C}$-linear subspace of $E$ and every $X \in \mathrm{g}$ is tangent to $V$.

PRoof. For every $X=\left(\alpha-q_{\alpha}(z, z)\right) \partial / \partial z \in \mathfrak{p}$ and $Y=\lambda(z) \partial / \partial z \in \mathfrak{f}$, we have

$$
[X, Y]=\left(\lambda(\alpha)-\lambda\left(q_{\alpha}(z, z)\right)+2 q_{\alpha}(\lambda(z), z)\right) \frac{\partial}{\partial z} \in \mathfrak{p},
$$

i.e. $\lambda(V) \subset V$ and $Y$ is tangent to $V$. In particular for $Y=Z=i z \partial / \partial z$, we get $i V=V$ and $q_{i \alpha}=-i q_{\alpha}$. Since $(\operatorname{ad} X)^{2} Z=4 i q_{\alpha}(\alpha, z) \in \mathfrak{f}$ and $\mathfrak{f}$ tangent to $V$, we have

$$
q_{\alpha}(\alpha, z) \in V \quad \text { for every } \alpha, z \in V .
$$

Substituting $\alpha+z$ for $\alpha$ in (9) we deduce that $q_{\alpha}(z, z) \in V$ for every $\alpha, z \in V$, i.e. $X$ is tangent to $V$. Q.E.D.

In [7] it has been proved that the group $G=$ Aut $(D)$ of all biholomorphic automorphisms of $D$ in a natural way is a real Banach Lie group. The connected component $G^{0}$ of $G$ is generated by the image of the exponential map exp: $\mathfrak{g} \rightarrow G$. Therefore $D \cap V$ is invariant under $G^{0}$ by Lemma 3. The mapping $\psi: G^{0} \rightarrow D \cap V$ defined by $g \rightarrow g(0)$ is analytic and the differential of $\psi$ at the point $\operatorname{id}_{D} \in G^{0}$ is precisely the topological isomorphism $\varphi: \mathfrak{p} \rightarrow V$ of Lemma 2. Therefore, by the implicit function theorem, the orbit $G^{0}(0)$ of the origin is open in $D \cap V$. Hence, by [5, Lemma 2], we have $G^{0}(0)$ $=D \cap V$. Let $\Omega=\left\{x \in D \mid G^{0}(x)\right.$ is a closed complex submanifold of $\left.D\right\}$. Obviously $0 \in \Omega$ and $\Omega$ is $G$-invariant since $G^{0}$ is normal in $G$. Let $x \in \Omega$ with $x \neq 0$. Then $W=\left\{t \in \mathbf{C}: t x \in G^{0}(x)\right\}$ is an analytic subset of the disc $\Delta=\left\{t \in \mathbf{C}:|t|<\|x\|^{-1}\right\}$.

Since $\{t \in \mathbf{C}:|t|=1\} \subset W$ we have $W=\Delta$ and therefore $0 \in G^{0}(x)$, or equivalently, $x \in G^{0}(0)$. But then

$$
\Omega \subset G^{0}(0)=D \cap V \subset G(0) \subset \Omega \text {, i.e. } \Omega=G^{0}(0)=G(0)=D \cap V \text {. }
$$


Proposition 1. Suppose $D$ is the unit ball of a complex Banach space $E$ and $G=$ Aut $(D)$ is the group of all biholomorphic automorphisms of $D$. Then $G$ is a real Banach Lie group with the following properties:

(i) The mapping $G \times D \rightarrow D$ defined by $(g, x) \mapsto g(x)$ is analytic.

(ii) $G=G^{0} G_{0}$ where $G^{0}$ is the connected component of $G$ and $G_{0}=\{g$ $\in G: g(0)=0\}$ is the isotropy subgroup at the origin.

There exists a closed $\mathbf{C}$-linear subspace $V \subset E$ such that

(iii) $G(0)=D \cap V=\{x \in D: G(x)$ is a closed complex submanifold of $D\}$ and $D \cap V$ is a bounded symmetric domain in $V$ on which the groups $G$ and $G^{0}$ act transitively.

Proof. Statement (i) is contained in [7] and (iii) has already been proved. To prove (ii) let $g \in G$. Then $g(0) \in G(0)=G^{0}(0)$, i.e. $g(0)=h(0)$ for some $h \in G^{0}$. But then $h^{-1} g \in G_{0}$, which proves the statement.

COROLlaRY. Every $g \in G$ admits a holomorphic extension onto an open neighborhood of $\bar{D} \subset E$ (with values in $E$ ).

Proof. If $g \in G_{0}$ this is true since $G_{0} \subset G L(E)$. If $g \in G^{0}$ this is true since $G^{0}$ is generated by $\exp (\mathfrak{g})$ and since every $X \in \mathfrak{g}$ is holomorphic on $E$. (Compare [4].)

Now we can prove the Theorem: Suppose $\tilde{E}$ is another complex Banach space with unit ball $\tilde{D}$ and $f: \tilde{D} \rightarrow D$ is a biholomorphic mapping. Then Proposition 1 also applies to $\tilde{D}$ and $\tilde{G}:=$ Aut $(\tilde{D})$. In particular, it follows from (iii) that $f(\tilde{G}(0))=G(0)$, i.e. there exists a biholomorphic map $h: \tilde{D}$ $\rightarrow D$ with $h(0)=0$. But then $h$ has to be linear. Q.E.D.

Remark. For the proof of Proposition 1 we used only that $D \subset E$ is a bounded balanced domain except in the proof of Lemma 2 (where the application of Harris' result also requires that $D$ be convex). Recently J. P. Vigue communicated to us without proof the following statement: For every bounded domain $D \subset E$ and every $a \in D$ the mapping $\mathrm{g} \rightarrow E \times L(E)$ defined by $h(z) \partial / \partial z \mapsto\left(h(a), h^{\prime}(a)\right)$ has closed image. From this, Lemma 2 and, hence, Proposition 1 would follow for every bounded balanced domain $D \subset E$.

AdDed IN Proof. The statement of Vigué appeared in J.-P. Vigué, C.R. Acad. Sci. Paris Sér. A 282 (1976), p. 113, Corollaire.

\section{REFERENCES}

1. J. Dieudonné, Foundations of modern analysis, Pure and Appl. Math. vol. 10, Academic Press, New York and London, 1969. (1960 ed., MR 22 \#11074.)

2. L. A. Harris, A continuous form of Schwarz's Lemma in normed linear spaces, Pacific J. Math. 38 (1971), 635-639. MR 46 \# 4167.

3. - Bounded symmetric homogeneous domains in infinite dimensional spaces, Proceedings on Infinite Dimensional Holomorphy, Lecture Notes in Math., vol. 364, Springer-Verlag, New York, 1973, pp. 13-40.

4. W. Kaup, Über das Randverhalten von holomorphen Automorphismen beschränkter Gebiete, Manuscripta Math. 3 (1970), 257-270. MR 43 \#3502.

5. - On the automorphisms of certain symmetric complex manifolds of infinite dimension, An. Acad. Brasil Ci. (to appear). 
6. L. Nachbin, Topology on spaces of holomorphic mappings, Springer-Verlag, New York, 1969. MR 40 \#7787.

7. H. Upmeier, Uber die Automorphismengruppen beschränkter Gebiete in Banachräumen, Thesis, Tübingen, 1975.

Department of Mathematics, University OF Kentucky, LeXington, KentuCKy 40506

Mathematisches Institut der Universität, 74 TÜBingen, Auf Der Morgenstelle 10, GermanY (Current address of W. Kaup and H. Upmeier) 